\title{
高校分析化学实验室废液的管理与处理
}

常静 $1,2,3$, 商闯 3 ,*

1 天津大学化学化工国家级实验教学示范中心, 天津 300072

2 天津大学化学化工国家级虚拟仿真实验教学中心, 天津 300072

3 天津大学理学院, 天津 300072

摘要: 化学实验会产生数量可观的实验废液, 这些废液如果处置不当, 可能会污染环境, 危害人们的身体健康。本文 以分析化学教学实验室为例, 分析了高校实验室废液的管理现状, 探讨了一些可行的管理和处理方法, 并提出了一些 有效减少实验室废液产生的建议, 为有类似问题的化学实验室提供了参考。

关键词: 分析化学实验室; 实验废液; 分类处理

中图分类号: G64; O6

\section{Management and Treatment of Liquid Waste in College Analytical Chemistry Laboratory}

\author{
Jing Chang ${ }^{1,2,3}$, Chuang Shang $3{ }^{3,}$ \\ ${ }^{1}$ National Demonstration Center for Chemistry and Chemical Engineering Education, Tianjin University, Tianjin 300072 , \\ China. \\ ${ }^{2}$ National Virtual Simulation Experimental Teaching Center of Chemistry and Chemical Engineering, Tianjin University, \\ Tianjin 300072, China. \\ ${ }^{3}$ School of Science, Tianjin University, Tianjin 300072, China.
}

\begin{abstract}
A large quantity of experimental waste liquid can be produced in the chemistry laboratory. If the liquid waste is handled improperly, it not only will pollute the environment, but also is harmful to our health. Taking the analytical chemistry laboratory teaching as an example, this paper analyzes the current situation of liquid waste management, discusses solutions of management and treatment, and puts forward suggestions to effectively reduce the production of liquid waste in laboratories, which provided reference materials for the chemical laboratory with similar problems.
\end{abstract}

Key Words: Analytical chemistry laboratory; Experimental liquid waste; Classificatory treatment

化学是以实验为基础的学科, 实验过程中会产生大量的废液。废液的种类繁多、成分复杂, 多 具有一定的挥发性、毒性或腐蚀性, 直接接触人体, 可能会引起刺激、过敏、疼痛等症状, 严重的 会引起中毒、灼伤甚至器官衰竭等永久性伤害 ${ }^{[1]}$ 。废液在存放的过程中, 因分类不合理, 运输不谨 慎, 存放不规范等原因造成不同性质的废液发生化学反应, 也会酿成可怕的事故。未经处理的废液 排放到自然环境中也会渗入到土壤里、混合到地下水、挥发到空气中, 对我们日常生活的环境造成 危害, 从而影响到我们的健康 ${ }^{[2,3]}$ 。因此, 化学工作者要提高环保意识, 建立切实可行、简单高效的

收稿: 2020-04-09; 录用: 2020-04-14; 网络发表: 2020-05-08

“通讯作者, Email: shangc307@tju.edu.cn

基金资助: 天津大学实验室建设与管理改革立项项目(LAB2018-B07) 
废液管理制度与处理方法, 排除废液安全隐患。这既是化学工作者应尽的义务, 也是不可推卸的责 任。

\section{1 高校实验室废液处置现状}

高校化学实验废液主要来自于科研实验室和教学实验室, 主要种类可大致分为无机废液、有机 废液、化学试剂空瓶等。我校对于实验室废液的管理十分重视, 专门制定了一系列制度文件和管理 措施, 校、院、实验室三级管理, 责任落实到人; 学校资产处依托网上化学品管理平台对实验室废 液进行台账登记, 使实验室的化学品从购买、使用到回收皆有迹可查; 根据化学品平台上登记的数 量, 学校定期组织废旧试剂回收工作, 由学院定时定点组织分类回收, 最后交给专业公司进行集中 处理。同时，校资产处配置了实验室废液储存柜、废旧试剂暂存柜等设备以应对废旧试剂暂存问题。

虽然在制度完善、数字化平台建设和暂存设备购置方面有充分的考虑, 但在废液处理过程中也 会出现一定的实际问题。目前天津市具有资质的废液处理企业仅有一家, 回收周期只能由公司决定 而不能根据实验室需求安排; 如果短期内产生的废液量较大, 则只能暂存在实验室内, 随着废液储 藏的时间变长, 随之而来的安全隐患和安全风险也日益凸显; 经济成本上, 企业处理废液的费用可 能比购买试剂所花的费用还要高, 导致实验室经费紧张是常态。

我校化学化工国家级实验教学示范中心承担着重要的实验教学任务, 拥有无机化学实验室、分 析化学实验室、物理化学实验室、有机化学实验室、综合实验室以及大型仪器室六个主要组成部分, 面向本校的 20 个专业、200 多个教学班开设化学和化工类基础实验课程 20 多门, 涉及的实验内容 近百项, 每学年总人时数达 30 万。如此高强度的实验教学任务, 每年都会产生体积庞大的实验废 液。由于高校在进行实验教学时, 学生流动性大、人数多、学生操作实验水平参差不齐、实验习惯 多有不同, 导致实验废液管理成为实验室安全管理的重要组成部分。

如果可以根据实验教学的特点提前做好课程的安排, 在相对集中的时间做好实验废液的分类回 收工作, 根据废液种类采取简单易操作且经济成本合理的方法及时进行处理, 不仅可以节约成本, 还能创造良好的实验环境，降低废液存储带来的风险，排除安全隐患。

\section{2 分析化学实验室废液的产生和特点}

高校各化学教学实验项目因课程特点不同, 产生的废液种类、性质各不相同, 废液量大且呈现 不规律性, 现以我校化学化工国家级实验教学示范中心的分析化学实验室为例说明实验教学废液的 产生和特点。

分析化学实验分为化学分析实验和仪器分析实验两大部分, 化学分析实验课程主要是以滴定实 验为基础, 使学生掌握定量化学分析实验的基本知识、基本操作技能、典型的分析方法和实验数据 处理方法, 树立 “量” 的概念等; 仪器分析实验主要是以各种大型分析仪器为基础, 使学生掌握基 础和现代分析化学技术, 具备分析问题、解决问题的能力等。两个实验课程产生的教学实验废液, 各自具有各自的特点。化学分析实验课程的废液主要为酸碱滴定实验所产生的酸性废液和碱性废液, 络合滴定实验所产生的金属离子废液, 以及氧化还原滴定实验所产生的强氧化剂溶液, 如高锰酸钾 溶液等。化学分析实验废液种类多、产量大, 但成分基本确定, 由实验设置而定。为方便学生回收 废液, 每个实验室在固定区域配备不同种类的废液桶, 但每个实验室配备的废液桶数量有限, 为不 影响下一个班级正常上课的废液储备, 废液桶满后, 须及时原位处理, 或转移至集中储备室, 并配 备上新的废液桶。仪器分析实验课程的废液主要为原子吸收光谱仪产生的金属离子废液, 气相色谱、 高效液相色谱、电化学工作站产生的有机废液, 以及其他仪器测试产生的无机废液, 还有自主创新 实验产生的不确定成分的废液。这部实验废液浓度相对较小, 产量也相对较少, 但是化学成分复杂, 化学危害更大。然而, 两个实验课程产生的教学实验废液也具有共同点, 如实验前后清洗玻璃仪器 所产生的洗涤废液, 产量大, 危害较小。 


\section{3 分析化学实验室废液的管理与处理}

分析化学实验室废液的管理要遵守一定的规则, 废液回收主要按照酸性试剂、碱性试剂、有机 试剂和无机试剂进行分类, 废液桶占有对应的不同颜色的标签; 在废液存放区, 用黄色警戒线进行 标记, 划分出专用废液收集和存放区, 并在废液容器下放置托盘, 以防废液浅出。废液的管理离不 开台账的记录, 天津大学资产处开发了专用的化学试剂管理平台, 试剂通过平台购买后就记录在管 理平台内, 随着实验的开展, 试剂在使用后, 及时在管理平台记录使用台账, 每一阶段的实验结束 后, 对应在平台将购买的试剂销账, 并记录产生的废液; 废液桶满之后存入废液存储区, 提交废液 回收申请, 等待学校安排统一回收。

分析化学实验室废液的处理需简单便捷，适合实验室环境操作，主要分为以下四个部分。

\section{1 废液的回收再利用}

对于废液的处理首先要考虑的是能否循环再利用, 这样既能减少药品的消耗, 节约资源, 又能 减少因废液处理带来的经济压力, 还可以减少对环境的污染。

\subsection{1 “变废为宝”, 充分利用实验产物}

化学分析实验与仪器分析实验相结合, 使用前面实验的产物作为后面实验的原料, 合理安排实 验次序, “变废为宝”, 不再简单地丢弃实验产物, 减少资源浪费, 避免环境污染。如在 “硫酸铜 的制备” 实验中, 由学生制备出来的硫酸铜晶体可以作为分析实验 “恒电流电解法测定铜含量” 的 原料, 而硫酸铜电解产生的大量酸性废液中含有微量的 $\mathrm{Cu}^{2+}$ 离子, 又可以作为 “原子吸收法测定废 液中铜的含量” 实验的原料, 使学生掌握原子吸收分光光度计测定溶液中微量或痕量金属元素的分 析技术。

\subsection{2 利用实验废液, 开展综合设计性实验}

传统的分析实验教学项目以经典实验为主, 实验内容比较陈旧, 实验方法相对单一, 多数为验 证性实验。这些实验的开展虽然能够使学生加深对理论知识的理解, 锻炼学生的操作技能, 但对培 养学生独立思考、开拓创新以及综合实践的能力还有所欠缺, 而利用实验室现有资源开展综合设计 性实验, 则有利于学生的全面发展。在教师的引导和指导下, 利用实验废液, 结合仪器分析实验中 学习的现代仪器分析技术, 由学生自己探索, 通过查阅文献、设计实验方案、组织实验、分析实验 数据、撰写实验报告的锻炼, 既培养了学生分析问题、解决问题的能力, 又提高了学生的实验积极 性。孟春霞老师 ${ }^{[4]}$ 为学生设计了一个利用火焰原子吸收光谱法测定废液中 $\mathrm{Cu} 、 \mathrm{~Pb} 、 \mathrm{Zn} 、 \mathrm{Cd}$ 含量的 开放实验; 汪军老师 ${ }^{[5]}$ 为学生设计了一个利用分光光度计和原子吸收分光光度计测定实验废液中 $\mathrm{Cr}(\mathrm{VI})$ 的含量的实验; 李增新老师 ${ }^{[6]}$ 将实验室废液回收列为综合性设计性实验课题, 引导学生尝试设 计实验对各类无机废液进行处理和回收; 秦建芳老师 ${ }^{[7]}$ 将含铜废液的处理、回收及检测设计为大型 综合性实验课题, 这些实验都为我们提供了很好的思路。

\section{2 掌握几种常见的实验废液处理方法}

与科研实验室不同, 分析化学教学实验室废液的产生由实验课程的安排决定, 时间相对集中, 经常是五到六个班级同时上课, 尤其是化学分析的滴定实验, 短时间内会产生很多废液。如果只是 单纯的收集废液, 便需要配置更多的废液回收桶, 大量的废液存放在教学课堂内会形成安全隐患, 增加安全风险。所以采取简单易操作的方法及时处理实验废液, 成为节约资源、消除隐患的首选方 式。

\subsection{1 酸性、碱性废液}

目前对酸碱废液处理的普遍方法是酸碱中和处理。可以在清楚废液成分的前提下将酸碱溶液分 开回收, 等到处理废液时先将废液混合, 用 $\mathrm{pH}$ 试纸或便携式 $\mathrm{pH}$ 计检测溶液至中性即可排放, 若 $\mathrm{pH}$ 不达标, 则继续加工业碱或浓酸进行中和处理, 直至废液呈中性。

\subsection{2 铅铋混合废液}

铅是剧毒金属, 铋更是稀缺资源, 价格较贵, 铅铋混合废液若不经处理直接排放, 不仅浪费资 
源, 更危害环境。向混合废液中加入过量 $\mathrm{Na}_{2} \mathrm{~S}$, 产生黑色 $\mathrm{PbS}$ 和 $\mathrm{Bi}_{2} \mathrm{~S}_{3}$ 沉淀。沉淀经分离、水洗后, 加入硝酸, 加热进行消解, 去除不溶物后, 将溶液稀释至一定体积即可循环使用。

\subsection{3 锰废液}

高锰酸钾是基础化学实验中常用的氧化剂, 自然也会产生大量的含锰废液。这类废液不经处理 直接排放, 可能随饮用水进入人体, 对神经系统产生毒害 ${ }^{[8]}$ 。实验室处理废液采用碱化除锰法处理 含锰废液。将碱加入含锰废液中, 调节废液 $\mathrm{pH}$ 至碱性, 废液中的 $\mathrm{Mn}^{2+}$ 转变成 $\mathrm{Mn}(\mathrm{OH})_{2}, \mathrm{Mn}(\mathrm{OH})_{2}$ 迅速氧化生成稳定的难溶物 $\mathrm{MnO}_{2}$, 从而达到去除 $\mathrm{Mn}^{2+}$ 的目的。

\section{3 浓度低于排放标准要求的废液}

实验过程中清洗玻璃仪器的洗涤液、水浴锅和真空循环水泵中的残液, 化学含量低于国家污水 综合排放标准 ${ }^{[9]}$ 要求, 可直接排放。在仪器分析实验中, 部分实验是对微量和痕量元素的测定, 这些 实验废液中所含元素浓度经测试若低于国家污水综合排放标准的, 可以直接排放。分类回收的废液 经集中处理后, 达到国家污水综合排放标准要求后, 也可直接排放。分析化学实验室常见污染物排 放标准见表 1 。

表 1 分析实验室常见污染物最高允许排放浓度

\begin{tabular}{cc}
\hline 分析实验室常见污染物 & 最高允许排放浓度 $c /\left(\mathrm{mg} \cdot \mathrm{L}^{-1}\right)$ \\
\hline 总铜 & 1.00 \\
总铅 & 1.00 \\
总锌 & 2.00 \\
总锰 & 2.00 \\
总铬 & 1.50 \\
六价铬 & 0.50 \\
总镉 & 0.10 \\
苯酚 & 0.30 \\
硝基苯 & 2.00 \\
\hline
\end{tabular}

\section{4 综合废液}

仪器分析实验所产生的有机废液主要为有机溶剂、有毒的芳香族化合物等, 如高效液相色谱使 用后产生的甲醇废液、紫外-可见分光光度计使用后产生的酚类化合物等。有机废液如果直接排放到 自然环境, 经过不断的累积、储存, 最后在生物体内富集, 会危害人体健康。自主创新实验所产生 的实验废液成分更加复杂, 难以预测。这些成分复杂的综合废液, 如果在实验室环境进行处理, 则 耗时长、效率低, 还有可能产生二次污染, 存在安全隐患, 因此, 对于这类废液在收集过程中应根 据废液性质做好分类工作, 贴好标签, 注明主要成分、咜存时间等信息, 确保废液桶的密闭存放, 储藏过程中做好管理工作，最后交由有资质的企业统一回收处理。

\section{4 分析化学实验室针对废液处理问题的其他解决途径}

\section{1 开展虚拟仿真实验}

我校化学化工虚拟仿真中心作为第一批国家重点虚拟仿真中心, 虚拟实验教学系统也在不断完 善, 该系统采用 ZBrush、Maya、MAX 建模技术对物体进行 3D 精准建模, 通过 Mudbox 等工具绘 制 3D 模型贴图, 最终通过 Unity 引擎运行仿真交互程序并启动仿真算法。这些新技术的引用, 可以 使学生产生身临其境的感受, 实现实验过程仿真、实验结果仿真, 从而达到实验教学目的。对于一 些化学药品毒性较大的实验和产生的废液难以处理的实验, 我们可以通过虚拟实验教学系统进行, 这样不但有利于节约原料药品, 更可以减少化学废液的产生, 降低对环境的污染。 


\section{2 合理设计实验内容, 开发微量实验}

实验设计过程中应充分考虑废弃物对环境的影响, 尽量避免剧毒试剂的使用, 在保证实验效果 的前提下, 优先以微型实验为主, 尽量减少药品和试剂的使用, 对许多涉及药品浓度的实验进行探 索和改进。如在 “有机化合物的紫外吸收光谱测定” 实验中, 实验的设计是个定性实验, 对溶液浓 度没有很高的要求, 而紫外吸收灵敏度却很高, 因此应配制较低浓度溶液进行实验, 节省药品的消 耗。

\section{3 教师在教学中向学生渗透环保意识}

对废液的管理不应单纯地考虑如何处理废液, 更应考虑废液产生的源头, 应该从实验教学开始, 规范学生的实验操作, 培养学生养成良好的实验操作习惯, 更有利于减少实验废液的产生。如学生 采用差减称量法通过电子天平称取 $0.1000-0.1500 \mathrm{~g}$ 药品时, 每次称量超重都需重新称量, 但已取出 的药品不能再次使用, 这便造成药品的浪费, 严格按照少量多次的称量方法就会避免称量超重的现 象; 在使用容量瓶配制标准溶液时, 不使用玻璃棒引流, 可能会导致部分溶质损失, 定容最后一步 时, 不使用胶头滴管滴加蒸馏水, 则容易加过刻度线, 造成浓度偏低, 溶液也需要从新配制, 同样 造成药品的浪费; 挥发性溶液若不及时盖上试剂瓶塞则会导致溶液浓度变化, 增加实验失败的概率, 从而产生更多废液。

\section{4 实验员在工作中提高环保意识}

实验员的工作贯穿整个实验过程, 其环保意识在废液管理上起着至关重要的作用。实验员应加 强责任心, 根据上课人时数, 掌控各种试剂和药品的配备量, 不能配的太少, 供不上课堂使用, 也 不能富余太多, 造成资源浪费; 针对易变质的药品, 实验员要根据实验需求把握药品使用的时间和 单次使用量, 做到现用现配; 在准备多种状态相似颜色相同的固体药品或溶液时, 可以使用不同颜 色的标签, 提醒学生注意, 避免学生称错取错, 从而减少废液的产生。

\section{5 结语}

废液的产生是化学实验过程中不可避免的, 如何通过管理和处理降低废液对环境的污染、减少 废液处理成本是我们需要不断探索的问题。通过准确掌握化学实验废液的处理方法、严格要求实验 过程、加强化学废液的监督管理, 合理进行回收, 实现节能减排, 进而提高化学科学为社会服务的 水平, 对建设绿色环保、可持续性发展的和谐社会具有重要的意义。

\section{参 考 文 献}

[1] 张学东, 张宜欣, 刘永红. 实验室研究与探索, 2012, 29 (3), 357.

[2] 康希, 姜勇, 李心爱. 广州化工, 2012, 40 (5), 174.

[3] 兰景风, 俞娥. 大学化学, 2016, 31 (8), 71.

[4] 孟春霞, 李雅蕾, 毛丽莉. 实验室科学, 2016, 19 (5), 30.

[5] 汪军, 尹延斌, 时爱菊, 孙华, 董光霞. 中国教外教育, 2014, No. 6, 74 .

[6] 李增新, 薛淑云, 王国明, 李辉. 实验室研究与探索, 2008, 27 (7), 105.

[7] 秦建芳, 谭俊民, 弓巧娟. 大学化学, 2016, 31 (11), 72.

[8] 段睿, 杨翠英, 马小隆. 实验室研究与探索, 2014, 33 (7), 297.

[9] 国家环保总局科技标准司. 污水综合排放标准(GB8978-1996)//中华人民共和国国家标准. 北京: 中国环境保护科技出版社, 1997. 